\title{
Thank you to all our manuscript reviewers in 2015
}

Jan Lubinski ${ }^{1}$, Rodney J. Scott ${ }^{2}$, Rolf Sijmons ${ }^{3}$ and Sarah M. Theissen ${ }^{4^{*}}$

\section{Contributing reviewers}

The editors of Hereditary Cancer in Clinical Practice would like to thank all our reviewers who have contributed to the journal in 2015.

Without the participation of skilful reviewers, no academic journal could succeed, and we are grateful to the committed individuals who have given their time and expertise to the peer review of manuscripts for Hereditary Cancer in Clinical

Practice. We look forward to your continued support in 2016.

\author{
Stefan Aretz \\ Germany
}

Gabriela Balogh

Argentina

Elizabeth Bancroft

UK

Morgan Butrick

USA

Berta Campos

Spain

John V Conaglen

New Zealand

Cezary Cybulski

Tadeusz Debniak

Poland

Pawel Domagala

Poland
Thilo Dork

Germany

Dagmara Dymerska

Poland

D Gareth Evans

UK

Thomas Hansen

Denmark

Shirley Hodgson

UK

Frans Hogervorst

Netherlands

Evgeny Imyanitov

Russia

Arvids Irmejs

Latvia

Anna Jakubowska

Poland

\author{
Wojciech Kluźniak \\ Poland
}

Kristina Lagerstedt Robinson

Sweden

\section{Marcin Lener}

Poland

\section{Annika Lindblom \\ Sweden}

Finlay Macrae

Australia

\section{Diptasri Mandal}

USA

\section{Alexandra Martins \\ France \\ Cliff Meldrum \\ Australia \\ Janusz Menkiszak \\ Poland}

* Correspondence: sarah.theissen@biomedcentral.com

'Department of Genetics and Pathomorphology, Pomeranian Medical

University, Szczecin, Poland

${ }^{2}$ Discipline of Medical Genetics, Faculty of Health, University of Newcastle

and Hunter Medical Research Institute, New South Wales, Australia

${ }^{3}$ Department of Genetics, University Medical Center Groningen, Groningen,

The Netherlands

${ }^{4}$ BioMed Central, 236 Gray's Inn Road, London WC1X 8HB, UK 
Caterina Mian

Italy

Pal Moller

Norway

Steven Narod

Canada

Jan Oosterwijk

Netherlands

\section{Carmen Radecki Breitkopf}

USA

Malgorzata Stawicka Nielacna Poland

Bente Talseth-Palmer

Australia

Amanda Toland

USA
Annemieke van der Hout

Netherlands

Rob van der Luijt

Netherlands

Michelle Wong-Brown

Australia

Xingnan Zheng

USA 